\title{
Erratum
}

\section{Erratum: Vinukonda et al., "Hyaluronidase and Hyaluronan Oligosaccharides Promote Neurological Recovery after Intraventricular Hemorrhage"}

In the article "Hyaluronidase and Hyaluronan Oligosaccharides Promote Neurological Recovery after Intraventricular Hemorrhage," by Govindaiah Vinukonda, Preeti Dohare, Arslan Arshad, Muhammad T. Zia, Sanjeet Panda, Ritesh Korumilli, Robert Kayton, Vincent C. Hascall, Mark E. Lauer, and Praveen Ballabh, which appeared on pages 872-889 of the January 20, 2016, issue, a grant number appeared incorrectly. "National Heart, Lung, and Blood Institute Grant HL104147 (M.E.L., V.C.H.)" should instead be "National Heart, Lung, and Blood Institute Grant HL113325 (M.E.L., V.C.H.)." The article has been corrected online.

DOI:10.1523/JNEUROSCI.0478-19.2019 